\title{
Polimorfismo entre poblaciones de Lutzomyia longipalpis, insecto transmisor de leishmaniosis visceral, sugiere la existencia de un complejo de especies
}

\begin{abstract}
Sandra Uribe
Resumen

Lutzomyia longipalpls se ha incriminado como el principal vector de leishmaniosis visceral en América latina y recientemente se ha asociado también con la transmisión de leishmaniosis cutánea en algunos países de Centroamérica. Por el carácter potencialmente fatal de la enfermedad que transmite, esta especie ha sido objeto de numerosas investigaciones que incluyen desde identificación taxonómica y control, hasta ecología, comportamiento y capacidad vectorial. Esta revisión recopila la literatura que hasta la fecha permite postular la existencia de Lu. longipalpis como un complejo de especies que podrían diferir en su capacidad vectorial con importantes implicaciones en la transmisión de la enfermedad.

Palabras clave: Lutzomyia longipalpis, Leishmaniosis, complejo de especies, Flebotomíne

Polymorphism in Populations of Lutzomyia longipalpis, Agent of Visceral Leishmaniasis, Suggests the Existence of a Species Complex

\section{Summary}

Lutzomyia longipalpis has been incriminated as the most important vector of visceral leishmaniasis in Latin America and recently it also has been associated with the transmission of cutaneous leishmaniasis in some Central American countries.

Because leishmaniasis is a potentially fatal disease $L u$. longipalpis has received great attention leading to the publication of several studies (including behaviour, vectorial capacity and taxonomy). This compilation includes the literature proposing the existence of Lu longipalpis as a species' complex consisting of at least three different species, suggesting important implications for leishmaniasis transmission.
\end{abstract}

Key Words: Lutzomyia longipalpis, Leishmaniasis, species' complex, Phlebotomines.

La leishmaniosis cuyo agente causal es el parásito tripanosomátido del género Leishmania, se considera como una de las enfermedades de mayor importancia en salud pública además de la malaria, la oncocercosis y la enfermedad de Chagas, todas ellas transmitidas por insectos hematófagos $(1,2)$.
Leishmania chagasi es el agente etiológico de la leishmaniosis visceral en Latinoamérica, una de las formas más graves de la enfermedad, considerada como potencialmente fatal, con aproximadamente 1.6 millones de personas que residen en áreas endémicas y 16.000 casos reportados anualmente (3-7).

Ciencias Básicas Biomédicas, Universidad de Antioquia (PECET-CDC) 
Los parásitos después de ser inoculados por la picadura del vector, se replican en los macró-fagos del hospedero, causando posteriormente alteraciones en la forma y función del hígado, el bazo, la médula ósea y los nódulos linfáticos (5).

$L u$. longipalpis es el principal vector de la leishmaniosis visceral y el incremento de esta forma de la enfermedad ha generado numerosas investigaciones, muchas de las cuales consideran el vector como blanco potencial para interrumpir la transmisión $(1,2)$.

Los estudios epidemiológicos y de control de la leishmaniosis que incluyen la identificación del vector, se dificultan por aspectos como el tamaño muy pequeño de los especímenes y su laboriosa manipulación, y por la presencia de complejos de especies o especies morfológicamente iguales que pueden presentar diferencias en comportamiento y capacidad vectorial, con importantes implicaciones en la epidemiología de la enfermedad $(6,8,9)$.

En el caso particular de Lu. longipalpis se presume la existencia de un complejo de especies evidenciado por los polimorfismos encontrados entre poblaciones a niveles morfológicos, fisiológicos e isoenzimáticos, y más recientemente a nivel molecular en la secuencia de nucleótidos del péptido MAXADILAN, un péptido vasodilatador que se encuentra en la saliva de este vector y que al parecer tiene influencia en la reacción eritematosa y el desarrollo de la infección (10).

Aunque las técnicas clásicas de identificación de insectos y algunas moleculares como la citogenética, los hidrocarbonos cuticulares y las isoenzimas, se han utilizado con algún éxito para estudiar los complejos de especies en los vectores de leishmaniosis, la obtención directa de secuencias de ADN, ofrece por primera vez la posibilidad de examinar y comparar transcritos de especímenes individuales, para complementar los estudios de genética de poblaciones en estos insectos $(11,12,13)$.

En consecuencia, la investigación sobre la variabilidad genética de los vectores de leishmaniosis está en su momento y las implicaciones de esta variabilidad en la epidemiología y control de la enfermedad, podrán evaluarse con mayor precisión $(6,14)$.

En esta revisión se presentan y analizan las evidencias que sustentan la existencia del complejo Lu. longipalpis incluyendo la obtención de secuencias de ADN mitocondrial, una técnica de gran utilidad en estudios de genética de insectos, que empieza a implementarse en el estudio de flebotomíneos y en Colombia en particular, en poblaciones de $L u$. longipalpis y $L u$. evansi, vectores de leishmnaiosis visceral $(13,15$ 17).

\section{Distribución geográfica}

Lu. longipalpis (Diptera: Psychodidae) fue descrita en 1912 por Lutz y Neiva a partir de especímenes colectados en Sao Pablo (Brasil) como una especie perteneciente al subgénero Lutzomyia (18).

Esta especie posee una gran distribución geográfica desde los $20^{\circ} \mathrm{N}$ en México hasta los $28^{\circ} \mathrm{S}$ en Argentina, incluyendo países como Guatemala, Costa Rica, Honduras, Colombia, Venezuela, Brasil, Paraguay y Bolivia. Sin embargo, está aparentemente ausente en la costa pacífica de Sudamérica en países como Ecuador, Perú o Chile donde no se conocen casos de leishmaniosis visceral $(3,8,14,19)$.

Lu. longipalpis se encuentra en zonas tropicales en una variedad de hábitats que comprenden desde áreas rocosas, áridas y semiáridas, hasta muy húmedas y boscosas en la rivera del río Amazonas (8,20-23) (Figura 1). Recientemente, se ha sugerido que cambios ecológicos y demográficos en América Latina, han propiciado la colonización exitosa de Lu. longipalpis en regiones donde no se había encontrado previamente y han facilitado la existencia de grandes poblaciones de este vector en sitios periurbanos (2).

En Colombia por ejemplo, aunque su distribución geográfica coincide con la parte superior y media del valle del río Magdalena y su presencia se asoció con zonas áridas y semiáridas, hace poco se encontró en Río Claro Antioquia, una zona típica de bosque húmedo tropical caracterizada 


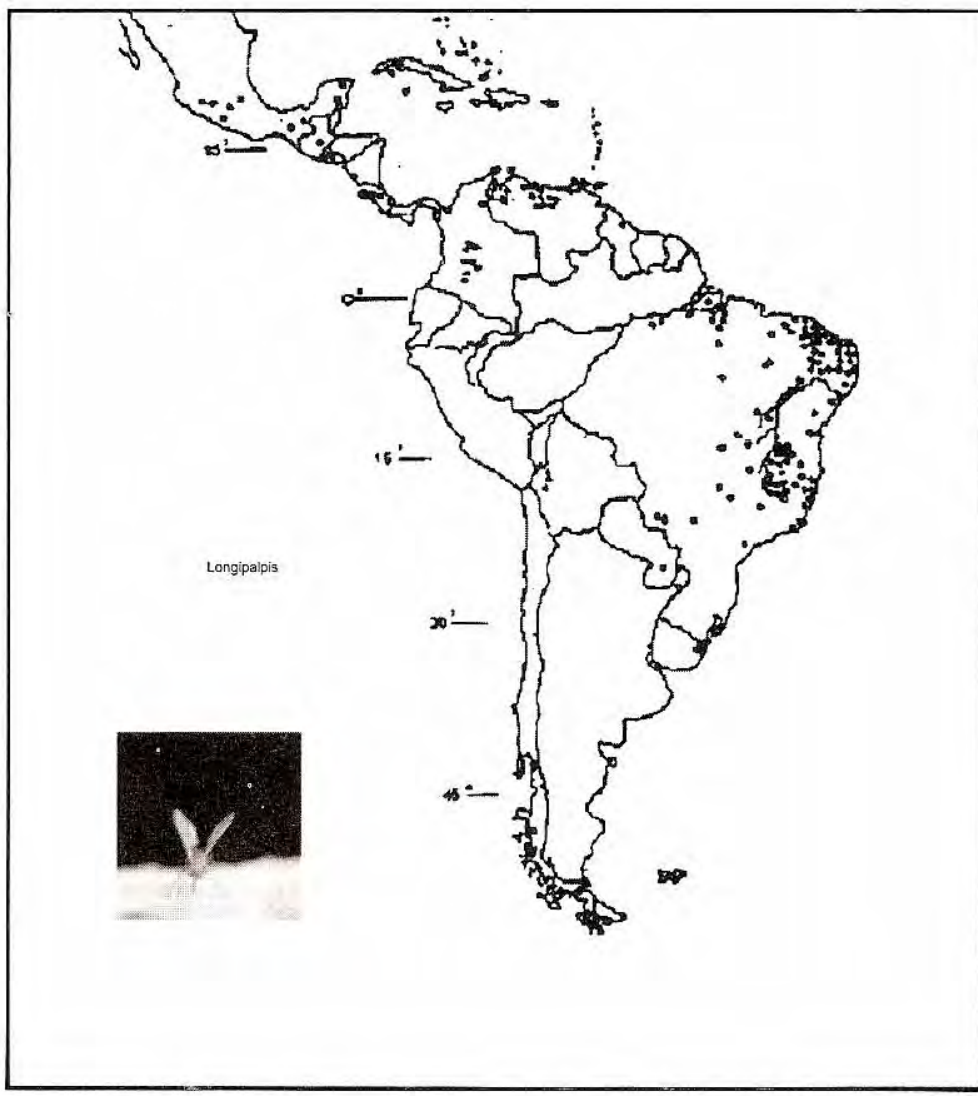

Figura 1. Distribución geográfica de Lutzomyja longipalpis en Latino América. (tomado de Young \& Duncan 1994).

por una vegetación exuberante, con altas temperaturas y régimen de lluvias que aumentan considerablemente la humedad relativa $(24,25)$; situaciones similares se describieron en Brasil Estado de Ceará y en un sitio periurbano, cerca de Rio de Janeiro (22).

Un aspecto interesante de la distribución de $L U$. longipalpis y su relación con la transmisión de la leishmaniosis visceral, es que en la actualidad no se sabe con certeza si este insecto se está expandiendo y colonizando áreas deforestadas en los alrededores de las ciudades neotropicales como resultado de su asociación con reservorios y humanos y de las modificaciones en el medio ambiente, o si su distribución discontinua se explica como remanentes de esta especie -con una distribución original continua- que fue separada por procesos climáticos y geológicos que ocasionaron aislamiento geográfico y disminución del flujo genético, facilitando así procesos de especiación (14).
La precisión sobre el origen y los patrones de distribución de Lu. longipalpis, aparece como uno de los estudios potenciales a realizar utilizando ADN mitocondrial, ya que por heredarse de forma materna, este ADN refleja los patrones genéticos en relación con la distribución geográfica (filogeográficos), facilitando la reconstrucción de los escenarios evolutivos, permitiendo además entender y relacionar la distribución del vector y del parásito en términos de coevolución, como recientemente fue realizado por Esseghir et al.1997 con vectores de Leishmania del viejo mundo $(11,13,15)$.

\section{Variabilidad morfológica}

La variabilidad morfológica en Lu. longipalpis fue descrita en 1969 por Mangabeira (26) a partir de especímenes capturados en Brasil. Esta variabilidad consiste dos o cuatro pares de manchas pálidas en los terguitos de los segmentos abdominales III y IV en los machos, así mismo se han observado algunos fenotipos intermedios 
como resultado de cruces entre individuos con los fenotipos originalmente descritos (Figura 2) (27).

No se ha encontrado una asociación directa entre el fenotipo y la transmisión de la leishmaniosis visceral, aunque originalmente se pensó que la forma con dos manchas tenía una participación más activa, ya que se encontraba en todas las zonas de transmisión de la enfermedad (27).

Variaciones morfológicas sutiles son frecuentes entre insectos de una misma especie, sin embargo, la asociación de los fenotipos con la producción de diferentes feromonas en $L u$. longipalpis, constituye una evidencia más sobre la existencia de un complejo de especies (6).

\section{Aislamiento reproductivo}

Los primeros intentos de cruce en laboratorio entre poblaciones de Lu. longipalpis fueron

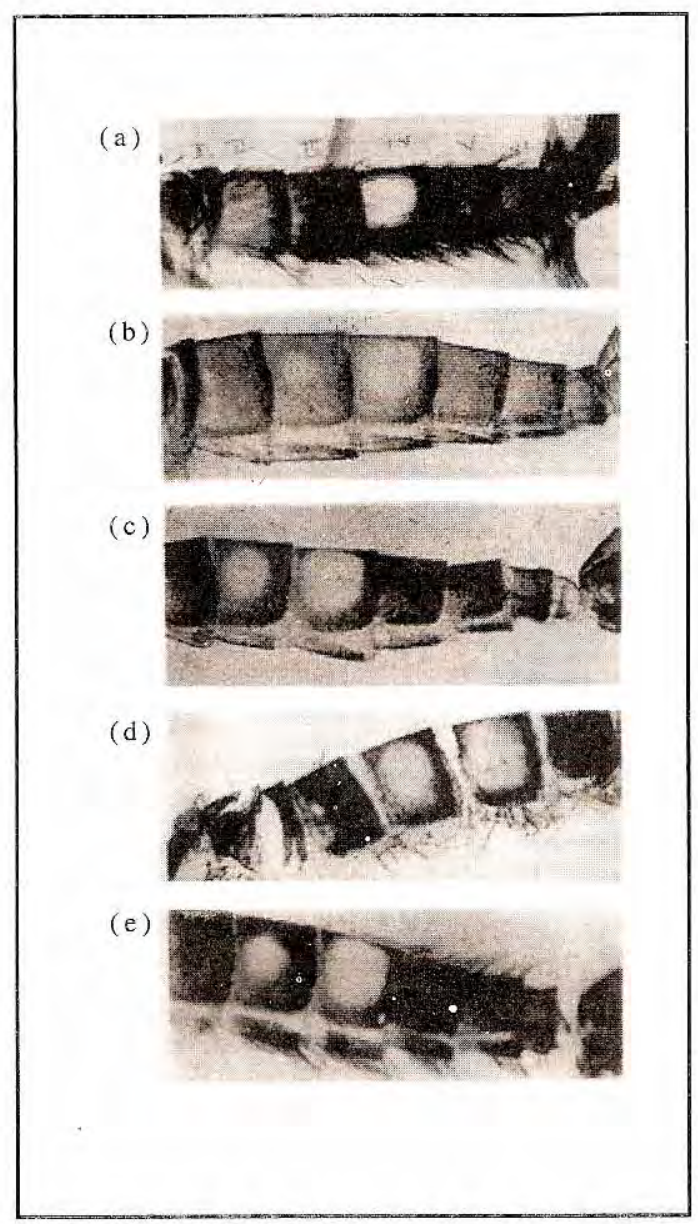

realizados después de observar diferencias en adaptabilidad a métodos de colonización en especímenes capturados en diferentes zonas geográficas de Brasil $(27,28)$.

Los cruces mostraron aislamiento reproductivo parcial entre poblaciones con uno y dos pares de manchas en los terguitos; sin embargo, se encontró evidencia contradictoria que disminuyó la posibilidad de identificar morfológicamente en los machos, dos especies de Lu. longipalpis (27).

Cruces de laboratorio entre especímenes provenientes de colonias de Colombia, Brasil y Costa Rica, mostraron esterilidad en la progenie de todos los machos, interpretada como indicador de especie para las formas parentales que produjeron descendencia estéril y junto con algunos estudios isoenzimáticos, estos resultados llevaron a los investigadores a proponer la existencia de por lo menos 3 especies geográficamente separadas conformando el complejo Lu. longipalpis, provenientes de estos países $(4,6)$.

Algunos estudios muy recientes indican que las diferencias isoenzimáticas entre $L u$. longipalpis provenientes de colonias, no corresponden necesariamente a diferencias genéticas presentes en las poblaciones del campo, ya que el proceso de colonización en sí, tiene efectos que pueden reflejarse en la variabilidad encontrada. Por lo tanto, cruces entre insectos de colonias realizados para definir la presencia y el número de especies del complejo Lu. longipalpis, necesitan ser verificados cuidadosamente con especímenes del campo $(29,30,31)$.

Figura 2. Variación morfológica en los terguitos de machos de Lutzomyia longipalpis. (tomado Ward et al. 1988).

(a) Una sola mancha en el segmento IV especímen proveniente de México.

(a) Una y media mancha en los segmentos III y IV especímen proveniente Paraiba, Brasil.

(b) Una y 3/4 mancha en los segmentos III y IV especímen proveniente Joao Pessoa, Brasil.

(c) Dos manchas en los segmentos III y IV especímen proveniente Morada Nova Ceará Brasil.

(d) Media mancha en segmentos II y IV de la progenie FI de cruces de laboratorio entre especímenes de una mancha (Isla de marajo) Pará, Brasil y dos manchas (Morada Nova, Ceará Brasil). 
No obstante, el trabajo con Lu. longipalpis de colonias de Brasil, Colombia y Costa Rica, fue realizado con colonias de origen reciente y se constituye en uno de los trabajos pioneros en el estudio y la determinación de Lu. longipalpis como un complejo de especies $(4,6,14)$.

\section{Fisiología y Comportamiento}

Los machos de Lu. longipalpis producen feromonas sexuales que presentan variabilidad entre poblaciones y parecen estar mediando el aislamiento reproductivo; las manchas pálidas presentes en los últimos segmentos abdominales de los machos se asociaron con la liberación de estas feromonas $(14,27,28,32,33)$.

Tres tipos de feromonas se han determinado en poblaciones de Brasil, tres en poblaciones de Honduras y uno en poblaciones de Costa Rica $(6,31,34)$. Sin embargo, no se ha establecido una relación directa entre el tipo de feromona producida por Lu. longipalpis, y las manifestaciones clínicas de la enfermedad.

En Centro América Leishmania chagasi causa no sólo leishmaniosis visceral sino también leishmaniosis cutánea atípica $(19,31,35)$ y en cada una de las zonas con diversas manifestaciones clínicas se han identificado poblaciones de $L u$. longipalpis que producen diferentes feromonas. Esto sugiere la presencia de varias especies que podrían modular el desarrollo de la enfermedad y explicar la variabilidad en las manifestaciones clínicas, ya que las cepas de L. chagasi aparecen idénticas cuando se analizan por las técnicas tradicionales $(6,31,34)$.

Estudios isoenzimáticos de Lu. longipalpis provenientes de zonas de Centroamérica con leishmaniosis visceral y cutánea atípica por $L$. chagasi, no revelaron la variabilidad sugerida prêviamente por las feromonas (31). Estos resultados contrastan con la teoría de Lanzaro et al. 1993 y Lanzaro y Warburg 1995 (4,6) según la cual, diferentes especies del complejo longipalpis -identificadas por la producción de feromonas y/ o isoenzimas - serían responsables de las diferencias encontradas en las manifestaciones clínicas de la enfermedad $(4,6,10)$.

Debe considerarse que en la leishmaniosis, características como el estado inmune del paciente pueden afectar significativamente las manifestaciones clínicas de la enfermedad, como se ha observado recientemente en pacientes con coinfección SIDA - Leishmania (36).

Dadas las discrepancias entre los datos de feromonas e isoenzimas y en ausencia de evidencia que lo confirme, sería apresurado explicar la variabilidad en la clínica de la enfermedad como resultado de la variabilidad genética del vector y no como la interacción de una serie de factores entre los cuales aparecen la respuesta inmune del paciente y la variabilidad genética del vector.

\section{Variabilidad isoenzimática y análisis morfometricos}

Las isoenzimas se han utilizado frecuentemente para estudiar las posibles especies del complejo Lu. longipaipis. Como se mencionó previamente, estos estudios revelan gran variabilidad entre poblaciones geográficamente separadas y con base en ellos se ha postulado la existencia de por lo menos tres especies del complejo provenientes de Brasil, Colombia y Costa Rica $(4,6,21,37)$.

La figura 3 muestra un dendrograma que relaciona algunas poblaciones Centro-americanas de $\mathrm{Lu}$. longipalpis con base en distancias genéticas ( $D$ y Nei) y promedios aritméticos (Sneath y Sorai ) $(31,38)$.

En el dendrograma se observa con base en el análisis de 10 isoenzimas que las poblaciones de Centroamérica son muy uniformes entre sí, pero considerablemente diferentes de las poblaciones de Colombia y Brasil (31).

Estudios isoenzimáticos también fueron realizados por Dujardin et al. 1997 (39) para estimar las relaciones genéticas entre $L u$. longipalpis con uno y dos pares de manchas en los terguitos, provenientes de Bolivia y otros países como Colombia y Brasil; en ellos se encontró que las diferencias genéticas poseen una relación directa con la distancia geográfica y que el modelo de "aislamiento por distancia" explica la distribución de las poblaciones estudiadas. Estos hallazgos concuerdan con los estudios de dispersión en Lu. longipalpis y otros vectores de Leishmania que señalan poca capacidad de vuelo en estos insectos y que 


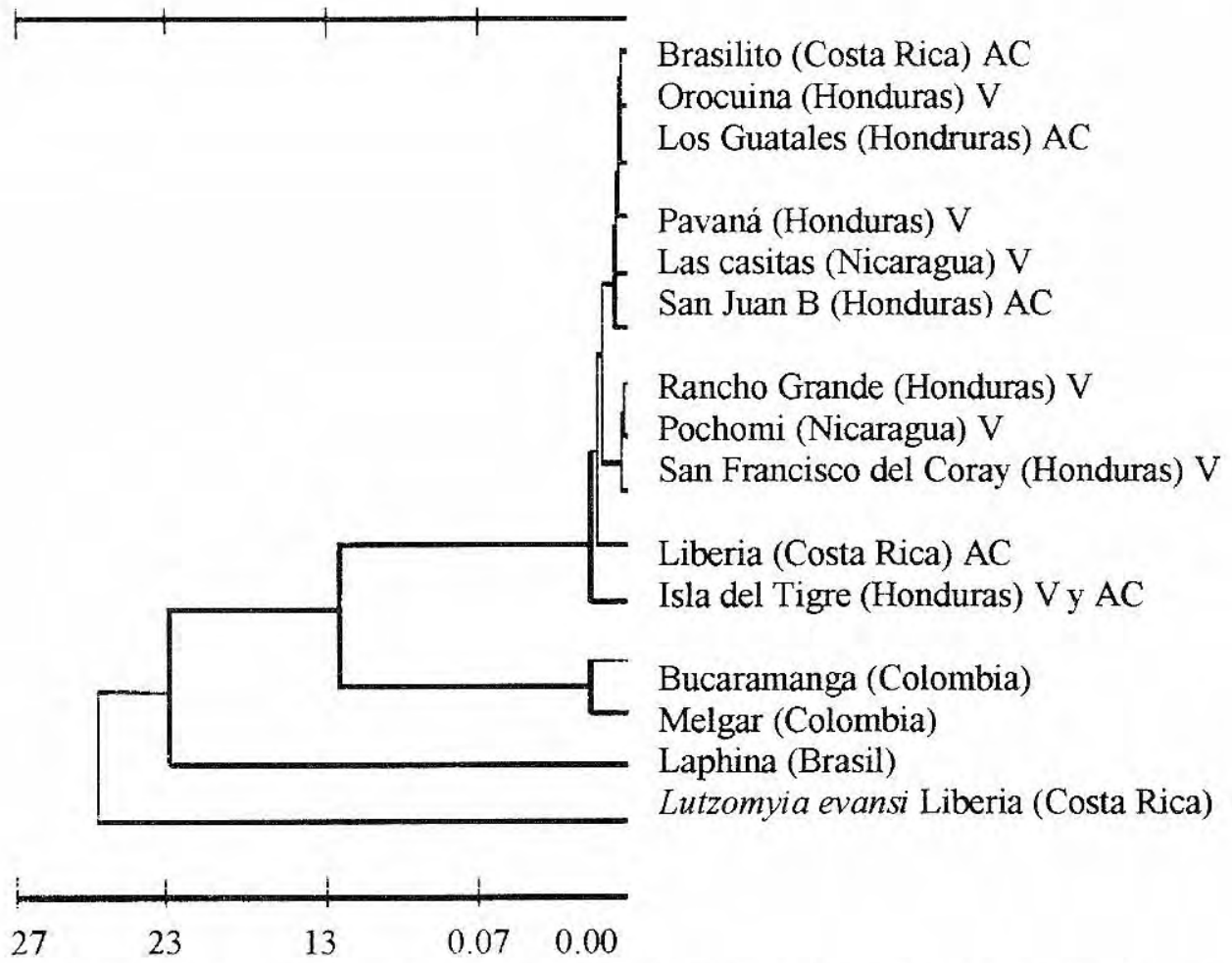

Figura 3. Dendrograma contruido con base en los valores de distancia genética (D) para poblaciones del campo de Lutzomyia logipalpis de Centro América y de colonia para Colombia y Brasil. Lu. evansi de Costa Rica fue incluida para comparación.

Las manifestaciones de la enfermedad relacionadas con las poblaciones de Centro América en las cuales se hizo énfasis, aparecen como:

$\mathrm{V}$ : Leishmaniosis visceral

AC: Leishmaniosis cutánea atípica

$\mathrm{U:}$ desconocida

Las de Sur América son todas viscerales, note la separación entre poblaciones de Centro y Sur América y Colombia y Brasil.

proponen las barreras geográficas como el sistema montañoso andino, como una de las causas principales de la divergencia y especiación ocurrida en Lu. longipalpis $(4,6,13,14)$.

Dujardin et al 1997 (39), también utilizaron morfometría de las alas para comparar poblaciones de $L$ u. longipalpis de Brasil, Colombia, Nicaragua y Honduras; la figura 4 muestra un dendrograma de UPGMA* construido con base en distancias genéticas (Mahglanobis) después de un análisis que incluyó la medición de cinco

\footnotetext{
* UPGMA: del inglés unweighted pair - group method using arithmetic averages: procedimiento de agrupación por pares usando medias aritméticas, método ampliamente utilizado en la taxonomía numérica (40).
}

caracteres en las alas; el análisis muestra las poblaciones de Colombia y Bolivia como grupos distintos, además, las poblaciones de Bolivia aparecen como dos agrupaciones que corresponden a los dos fenotipos presentes en este país (una y dos manchas en los terguitos) y las poblaciones de Nicaragua y Brasil aparecen como un grupo adicional. Las poblaciones de Bolivia y Colombia fueron asumidas como la misma especie de Lu. longipalpis de Colombia descrita inicialmente mediante isoenzimas por Lanzaro et al 1993 (4). Sin embargo, la agrupación entre las poblaciones de Nicaragua y Brasil se explicó por errores en la metodología (39).

Igualmente por isoenzimas Munsterman et al. 1998 (29) estudiaron la variabilidad genética de 


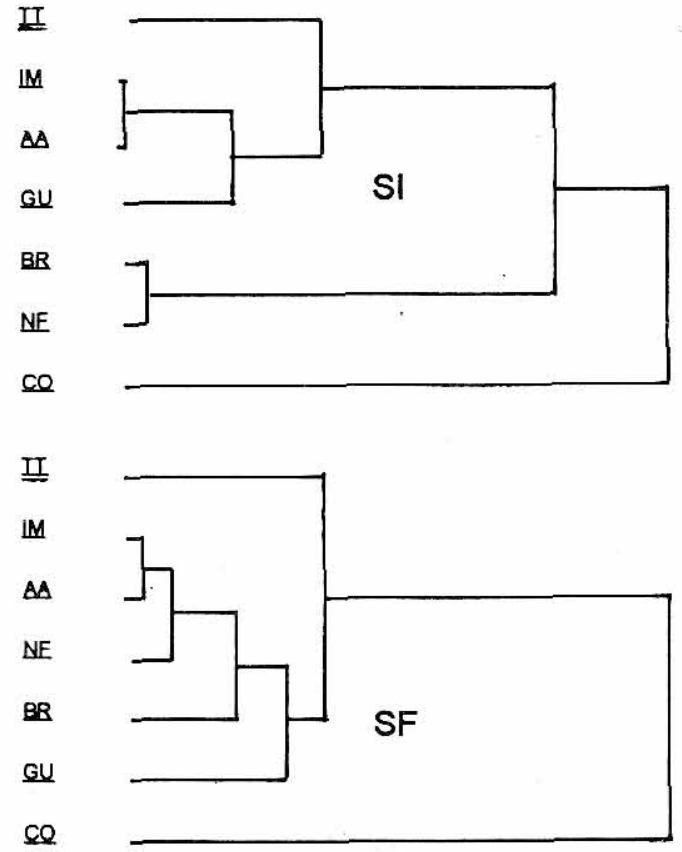

Figura 4. Dendrograma de UPGMA derivado de las distancias de Manghlanobis, los análisis fueron realizados teniendo en cuenta el tamaño de las alas SI (parte superior) y sin considerarlo SF (parte inferior). Los especímenes utilizados incluyeron 7 muestras de Lutzomyia longipalpis machos, de las localidades de Apa Apa, Imanaco y Guayaba, La Paz Bolivia (AA, IM, GU) Nicaragua cerca al límite con Honduras (NF) Minas Gerais Brasil (BR) y Colombia (CO), insectario de CIDEIM presumiblemente EI Callejón Cundinamarca igualmente Toro Toro (TT) Potosí, Bolivia. Los nombres de las localidades subrayados con una y dos líneas significan fenotipos con una y dos manchas en los terguitos respectivamente (tomado de Dujardin et al. 1997).

poblaciones colombianas de Lu. longipalpis en el valle del río Magdalena, encontrando altos niveles de flujo genético entre poblaciones cercanas y mínima subestructuración. Estos resultados confirman que el aislamiento genético aumenta con las distancias geográficas en Lu. longipalpis y este es uno de los pocos estudios que relaciona la variabilidad genética con la ecología y distribución del vector en sitios de transmisión como El Callejón, Cundinamarca quizá, el foco de transmisión de Leishmaniosis visceral mejor estudiado hasta ahora en Colombia $(14,21,29)$.

El número de especies de Lu. longipalpis determinadas por análisis isoenzimáticos y producción de feromonas varía grandemente, señalando la necesidad de estudios adicionales que permitan resolver estas discrepancias y estimar la verdadera naturaleza del complejo en relación con las modificaciones humanas del medio ambiente y las manifestaciones clínicas de la enfermedad.

\section{Variabilidad a nivel molecular}

Warburg et al. 1994 (10) investigaron la influencia de la saliva de Lu. longipalpis en el curso de la infección por $L$. chagasi en especímenes de Centro y Sudamérica, en especial con relación al péptido MAXADILAN, un péptido aislado de la saliva de Lu. longipalpis con una potente actividad vasodilatadora y que al parecer tiene gran influencia en el crecimiento y desarrollo de los parásitos, una vez inoculados por la picadura del vector (10).

Los resultados de este estudio señalaron que la saliva de los vectores de Brasil y Colombia posee mayor cantidad de MAXADILAN y en consecuencia una mayor actividad vasodilatadora en relación con los de Costa Rica, que presumiblemente son los vectores de la forma cutánea no ulcerativa, los cuales presentaron menor cantidad de MAXADILAN pero propiciaron la aparición y rápido desarrollo de lesiones cutáneas $(6,10,14)$.

Las diferencias en MAXADILAN aislado de Lu. longipalpis de estos países fueron comprobadas también a nivel molecular, utilizando un sistema de electroforesis (Polimorfismo Conformacional de Cadena Simple) que permite detectar indirectamente los polimorfismos en el ADN del péptido. Las diferencias en el ADN soportaron a nivel molecular las diferencias observadas en la cantidad y actividad del péptido que presumiblemente regularía el desarrollo de la enfermedad $(6,10,14)$.

Las variaciones encontradas de forma indirecta por la electroforesis en el péptido MAXADILAN, podrían verificarse mediante el análisis directo de las secuencias del gen que lo codifica, en especial para determinar si los cambios en las secuencias de Lu. longipalpis provenientes de zonas con diferentes formas de la enfermedad, 
ocurren en sitios del gen que están implicados en la función del péptido y por lo tanto, pueden correlacionarse con las diferencias encontradas en la capacidad del mismo para causar el eritema y propiciar el desarrollo del parásito en los primeros estados de la infección.

Recientemente otras técnicas moleculares han empezado a implementarse para interpretar el polimorfismo de Lu. longipalpis y entender su relación con la enfermedad. Estas técnicas incluyen la utilización de enzimas de restricción que cortan el ADN en sitios específicos generando diferentes patrones de bandas en electroforesis de ADN, características de especies (40) y la comparación directa de secuencias de ADN mitocondrial entre poblaciones geográficamente separadas.

Los genes utilizados incluyen la subunidad Dehidrogenasa 4 (NADH4), Citocromo Oxidasa II (COII) y la unidad larga ribosomal (LRU) (17, 41, Dotson et al 1998 com.pers.).

Igualmente, genes mitocondriales como el Citocromo b, el NADH1 y el RNA de transferencia ${ }^{\text {ser }}$ se han utilizado con éxito en estudios biosistemáticos de Lu. evansi y Lu. whitmani en Colombia y Brasil $(13,15,16)$.

Los estudios con ADN mitocondrial han confirmado la existencia del complejo $L u$. longipalpis separando las poblaciones de Colombia, Brasil y Costa Rica y corroborado las diferencias entre las poblaciones de Centro y Sudamérica descritas previamente por isoenzimas.

Los resultados señalan cambios significativos entre las secuencias de estos países (60-63 sustituciones nucleotídicas) y bajos niveles de flujo genético cuando se utilizan parámetros de genética de poblaciones como $\mathrm{Nm}$ (0.08-0.1) $(17,41,42)$.

Adicionalmente, estos estudios concuerdan con los análisis isoenzimáticos de Mutebi et al. 1998 (31), ya que en términos de las secuencias de ADN mitocondrial las poblaciones Centroamericanas no presentan diferencias significativas entre ellas (máximo 10 sustituciones nucleotídicas entre poblaciones de Costa Rica, Guatemala y Honduras), descartando una vez más la posibilidad de la existencia de dos especies de Lu. longipalpis en Centroamérica, que expliquen la presencia de leishmaniosis visceral y cutánea atípica.

El análisis filogenético con base en el gen NADH4 coincide en señalar la variabilidad previamente descrita por feromonas en Lu. longipalpis de Brasil, sugiriendo la presencia de por lo menos tres especies del complejo en este país (14).

La variabilidad en términos de sustituciones nucleotídicas observada entre especies morfológicamente diferenciables como $L u$. gomezi, Lu. bifoliata y Lu. evansi incluídas como control en los estudios de ADN mitocondrial es similar a la encontrada entre Lu. longipalpis de diferentes regiones de Centro y Sudamérica. De la misma forma, la variabilidad intraespecífica en los controles fue mucho menor al compararla con la de Lu. longipalpis como ocurre con los datos isoenzimáticos $(41,43,44)$; estos datos sugieren un proceso de especiación alopátrica.

Un aspecto de gran interés en el análisis del gen NADH4 es que la población de Lu. longipalpis de Girón Santander aparece sorprendentemente distinta de las demás poblaciones de Colombia, Venezuela y Brasil (41 sustituciones nucleotídicas vs 3-4 entre poblaciones de Cundinamarca y Huila) confirmando que la barrera de los Andes y otras barreras de tipo geográfico y climático, constituyen puntos críticos en la dispersión de Lu. longipalpis y sugiriendo con base en el concepto de especie filogenético y evolutivo, que en Colombia, existen por lo menos dos especies del complejo Lu. longipalpis.

A pesar del gran polimorfismo que sugiere la existencia de un complejo de especies en $L u$. longipalpis, el número de especies que lo conforman, su distribución y papel definitivo en la transmisión de la enfermedad están aún por definir, en especial si se consideran las discrepancias entre datos como los isoenzimáticos y de producción de feromonas. 
La hipótesis sobre la variación de esta especie no ha sido probada mediante transectos que involucren exclusivamente poblaciones del campo y en consecuencia, ésta aparece como una alternativa que podría explicar los datos encontrados hasta la fecha.

Cuando se consideran alternativas como los métodos basados en ADN para el estudio de complejos de especies como el de Lu. longipalpis, aparecen ventajas sobre otros métodos; por ejemplo, las mediciones se realizan sobre el genotipo más que sobre el fenotipo, se pueden seleccionar una o más secuencias de diferentes regiones y se pueden obtener y preparar muestras a partir de pequeñas cantidades de tejido, esto permite incluir especímenes muy pequeños, difíciles de abordar por taxonomía clásica. Además, los insectos pueden estar secos o preservados en alcohol o isopropanol $(40,43,44)$.

Sin embargo, cuando se trata de técnicas moleculares aparecen aspectos como el alto costo y la necesidad de personal especializado para la implementación de dichas técnicas. No obstante, se espera que en el futuro con la simplificación de algunas técnicas, el mejoramiento de otras y el desarroilo de secuencias de ADN diagnósticas para complejos de especies, la tecnología del ADN podrá alcanzar su máximo potencial en el campo de la entomología médica para confrontar, complementar o validar los hallazgos previos sobre el vector que se reflejan en la epidemiología de la enfermedad, como en el caso del complejo $L u$. longipalpis.

\section{Agradecimientos}

Al Doctor Charles Porter del CDC de Atlanta por su asesoría y dirección en el trabajo molecular con $L u$. y del cual esta revisión forma parte. El trabajo con $L u$. longipalpis ha sido realizado gracias al apoyo del Instituto Colombiano para el Desarrollo de la Ciencia y la Tecnología Francisco José de Caldas (COLCIENCIAS).

\section{Referencias}

1. World Health Organization. Control of the Leishmaniases: Technical Report Series 1990; 793:3-158.
2. Lainson R. Demographic changes and their influence on the epidemiology of the American Leishmaniasis. In M.W. Service (ed), Demography and vector-borne diseases. CRC, Boca Ratón, FL, 1989: 85-106.

3. Desjeux P. Human Leishmaniasis: Epidemiology and public health aspects. World Health Estatistics Quaterly 1992; 45:267-75.

4. Lanzaro G, Ostrovska K, Herrero M, Lawyer P, Warburg A. Lutzomyia longipalpis a species complex: Genetic divergence and interspecific hybrid sterility among three population. Ann of Trop Med Hyg 1993;48: 839-47.

5. Vélez ID, Agudelo S. Manual de procedimientos para el diagnóstico de Leishmaniosis cutánea americana. Editorial Universidad de Antioquia; 1996:35.

6. Lanzaro G, Warburg A. Genetic variability in phlebotomine sandflies: Possible implication for Leishmaniosis epidemiology. Parasitology Today 1995; 4: 151

7. Ashford RW, Desjeux P, deRaadt P. Estimation of population at risk of infection and number of cases of Leishmaniasis. Parasitology Today 1992; 8:104-5.

8. Young DG, Duncan MA. Guide to identification and geographic distribution of Lutzomyia sandflies in Mexico. The West Indies, Central and South America (Diptera: Psychodidae). Associated published Am Entomol Inst. Gainesville, 1994; 54: 881 .

9. SteinerWWM. Parasitization and speciation in mosquitoes: a hypothesis. In: R Pal, Kitzmiller \& T kanda (Eds). Cytogenetic and genetics of vector. Proceedings of a symposium of the XVIth Congress of Entomology. Kiodansha Ltd. Tokyo: Elsevier Biomedical 1981.

10. Warburg A, Saraiva E, Lanzaro G, Titus R, Neiva F. Saliva of Lutzomyia longipalpis sibling species differs in its composition and capacity to enhance Leishmaniasis. Phil. Franc. R. S. Ion 1994; 3455 (312): 223-30

11. Beard CB, Mills Hamm D, Collins FH. The mitochondial genome of the mosquito Anopheles gambiae: DNA sequences organization, and comparisons with mitochondrial sequences of other insects. Insect Molecular Biology 1993; $2: 103-24$

12. Feliciangeli DM, Castejon OC, Limongi J. Egg surface ultrastructura of eight New World phlebotomine sandfly species (Diptera: Psychodidae). J Med Entomol 1993; $30: 651-56$

13. Lerma JM. The biology of visceral Leishmaniasis vectors in the San Andrés de Sotavento focus, Colombia. University of London, 1996:163.

14. Bruce A, Mutebi JP, Lanzaro GC, Ward RD lamilton JGC. Current status of the Lutzomyia longipal 3 species complex. Men Inst Oswaldo Cruz. 1998:31-3.

15. Esseghir S, Ready PD, Killick-Kendrick BR Mitochondrial haplotypes and phylogeography of phlebr smus vec- 
tors of Leishmania major. Insect Molecular Biology 1997; 6: $211-25$

16. Ready PD, Day JC, Souza AA, Rangel EF, Davies CR. Mitochondrial DNA characterization of populations of Lutzomyia whitmani (Diptera: Psychodidae) incriminated in the peridomestic transmission of Leishmania species in Brazil (Submited).

17. Uribe S, Charles P, Vélez ID. Amplificación y obtención de secuencias de rRNA mitocondrial en Lutzomyia spp(Diptera: Psychodidae)vectores de Leishmaniosis. SOCOLEN 1998; 24:169-78.

18. Lutz A, Neiva A. Contribucao para o cohencimento das especies do genero Phlebotomus existentes no Brazil. Mem Inst Oswaldo Cruz. 1912; 4: 84-95.

19. Zeledón R, Murillo J, Gutierrez H. Observaciones sobre la ecología de Lutzomyia longipalpis (Lutz \& Neiva, 1912) y posibilidades de existencia de Leishmaniasis visceral en Costa Rica. Mem Inst Oswaldo Cruz 1984; 79:455-59.

20. Gallego J, Vélez ID. Presencia en la Isla Fuerte, Bolivar de Lutzomyia evansi vector de Leishmaniosis visceral. IATREIA (Medellín) 1995; 7:33-35.

21. Ferro C. Morrison AC, Torres M, Pardo R. Wilson ML, Tesh RB. Species composition and relative abundance of sand flies of the genus Lutzomyia (Diptera:Psychodidae) at an endemic focus of visceral Leishmaniosis in Colombia. J Med Entomol 1995; 32: 527-37.

22. Lainson R, Shaw JJ, Ryan L, Ribeiro RSM, Silveira FT. Leishmaniasis in Brazil:XXI. Visceral Leishmaniasis in the Amazon Region and further observations on the role of Lutzomyia longipalpis (Lutz \& Neiva, 1912) as the vector. Trans Roy Soc Trop Med Hyg 1985; 79:223-26.

23. Lainson R, Shaw JJ, Silveira FT, Fraiha H. Leishmaniasis in Brazil: XIX. Visceral Island of Marajó, Pará State. Trans Roy Soc Trop Med Hyg 1983; 77:323-30.

24. EspinalTLS, Montenegro ME. Formaciones vegetales de Colombia. Instituto Geográfico Agustin Codazzi: Bogotá, 1963.

25. LopezY, Osorio L, Alvarez G, Rojas J, Jiménez F, Gomez C. Sandfly Lutzomyia longipalpis in a cutaneous Leishmaniasis focus in central Colombia. Mem Inst Oswaldo Cruz, Rio de Janeiro, 1996; 9: 415-19.

26. Mangabeira $\mathbf{O}$. Sobre a sistematica e biologia dos phlebotomus do ceará. Bras Malaria Doencas Trop 1969; 21: 3-26.

27. Ward R, Phillips A, Burnet B, Marucondes C. The Lutzomyia longipalpis complex: Reproduction and distribution. Biosystematic of Hematophagous insect. In M. W Service (ed). Clarendon Press Oxford, 1988:257-69.

28. Lane R, Ward R. The morphology and possible function of abdominal patches in males of two forms of the
Leishmaniosis vector Lutzomyia longipalpis. Cahiers ORSTOM Sér Entomol Méd Parasitol 1984;22: 245-49.

29. Munsterman LE, Morrison AC, Ferro C, Pardo R, Torres M. Genetic structure of local population of Lutzomyia longipalpis (Diptera: Psychodidae) in Central Colombia. J Med Entomol 1998; 35: 82-9.

30. Mukhopadhyay J, Rangel E, Ghosh K, Munsterman L. Patterns of genetic variability in colonized strains of Lutzomyia longipalpis (Diptera: Psychodidae) and its consequences. Am J Trop Med Hyg 1997; 57: 216-21.

31. Mutebi JP, Rowton M, Herrero MV, Ponce C, Belli A et al. Genetic variability among population of the sand fly Lutzomyia longipalpis (Diptera: Psychodidae) from Central Am J Med Entomol 1998; 35:170-74.

32. Ward R, Ribeiro Ä, Ready P, Murthag A. Reproductive isolation between different forms of Lutzomyia longipalpis (Lutz \& Neiva 1912) (Diptera: Psychodidae) the vector of Leishmania donovaniChagas/Cunha \& Chagas and its significance to kala-azar distribution in South America. Mem Inst Oswaldo Cruz 1983; 78: 269-80.

33. Lane R, Phillips A, Molyneux D, Drocten G, Ward R. Chemical analyses of the abdominal glands of two forms of Lutzomyia longipalpis: Site of possible sex pheromone? Ann Trop Med Parasitol 1985; 79:225-29.

34. Hamilton JG, Ward RD, Dougherty M, Maignon JR, Ponce C, Ponce E. Comparison of the sex pheromone components of Lutzomyia longipalpis (Diptera: Psychodidae) from areas of visceral and typical cutaneous Leishmaniasis in Honduras and Costa Rica. Ann Trop Med Parasitol 1996; 90: 535-41.

35. Zeledón R, Hidalgo H, Viquez A \& Urbina A. Atypical cutaneous Leishmaniasis in a semiarid region of north-west of Costa Rica. Trans Roy Soc Trop Med Hyg 1989; 83:786.

36. Pratlong F, Dedet JP, Marty P, Portús M, Deniau M, Dereure J, et al. Leishmania-human immunodeficiency virus coinfection in the Mediterranean Basin: Isoenzymatic characterization of 100 isolates of the Leishmania infantum complex. Journal of Infectious Diseases 1995; 172:32326.

37. Bonnefoy S, Tybayrenc M, Le Pont F, Dujardin JP, Desjeux P, Ayala FJ. An isozymic study of Lutzomyia longipalpis (Diptera: Psychodidae), vector of visceral leishmaniasis in the "Yungas" (Bolivia). Cahiers ORSTOM Sér Entomol Méd Parasitol 1986; 24:213-17.

38. Nei M. Estimation of average heterozygosity and genetic distance from a small number of individuals. Genetics 1978; 89:583-90.

39. Dujardin JP, Torrez EM, Le Pont F, Hervas D. Isozymic and metric variation in the Lutzomyia longipalpis complex. Medi Vet Entomol 1997; 1:394-400.

40. Quicke DLJ. Principles and techniques of contemporary taxonomy. Blackie Academy \& Professional 1993.

41. Uribe S. Utilización del DNA mitocondrial para estudiar las relaciones filogenéticas en insectos vectores de Ma- 
laria y Leishmaniosis: Complejos de especies Anopheles maculipennis y Lutzomyia longipalpis. Facultad de Medicina Universidad de Antioquia- Center for Diseases Control CDC Atlanta, 1999.

42. Futuyma DJ. Evolutionary biology. Third edition. Sinauer Associates, Inc. Publishers Sunderland, Massachusetts, 1998:763.
43. Hill SM, Crampton M. DNA-based me lods for the identification of insect vectors. Ann Trop $\mathbf{N}$ :d Parasitol 1994; 88:3227-250.

44. Avise JC. Ten unorthodox perspectives on evolution prompted by comparative population genetics findings on mitochondrial DNA. Annual Review Genetics 1991;25:45-69. 